\title{
Vesicle adhesion and microemulsion droplet dimerization: Small bending rigidity regime
}

Cite as: J. Chem. Phys. 111, 7062 (1999); https://doi.org/10.1063/1.479998

Submitted: 23 April 1999 . Accepted: 26 July 1999 . Published Online: 05 October 1999

Edgar M. Blokhuis, and Wiebke F. C. Sager

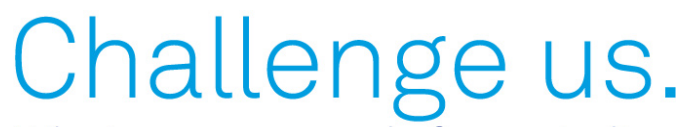

What are your needs for periodic

signal detection?
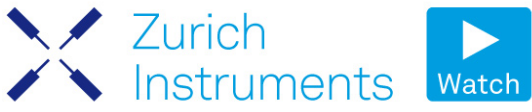

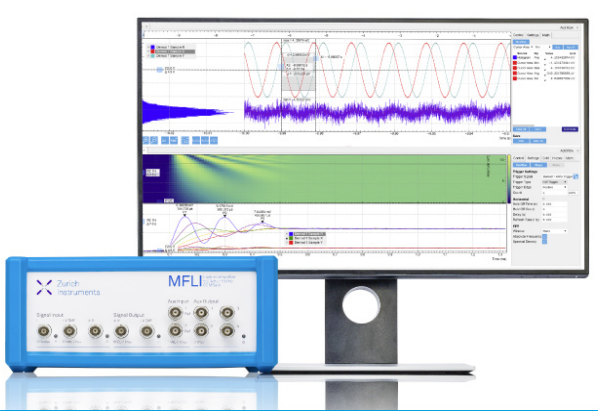

111, 7062 


\title{
Vesicle adhesion and microemulsion droplet dimerization: Small bending rigidity regime
}

\author{
Edgar M. Blokhuis ${ }^{\text {a) }}$ \\ Colloid and Interface Science, Leiden Institute of Chemistry, Gorlaeus Laboratories, P.O. Box 9502, \\ 2300 RA Leiden, The Netherlands \\ Wiebke F. C. Sager \\ Faculty of Chemical Technology, Membrane Technology, University of Twente, P.O. Box 217, \\ 7500 AE Enschede, The Netherlands
}

(Received 23 April 1999; accepted 26 July 1999)

\begin{abstract}
To study the vesicle-substrate unbinding transition and the onset of microemulsion aggregation, we calculate the curvature free energy of a vesicle adhered to a substrate and of two microemulsion droplets forming a dimer. Analytical expressions are derived in the small bending rigidity regime in which the length $(k / \sigma)^{1 / 2}$, constructed from the rigidity constant of bending $k$ and surface tension $\sigma$, is small compared to the typical size of the vesicle (droplet), $(k / \sigma)^{1 / 2} \ll R$. The leading contribution to the curvature free energy is shown to be proportional to $k^{1 / 2}$. The formulas derived are used to understand the experimentally observed aggregation of microemulsion droplets occurring in the direction of vanishing spontaneous curvature. In this way we intend to bridge the gap between the liquid state theories used to describe aggregation processes in microemulsion systems and the bending energy concept originally introduced by Helfrich to describe vesicles shapes and fluctuations as well as phase diagrams of microemulsion systems. (C) 1999 American Institute of Physics. [S0021-9606(99)71739-3]
\end{abstract}

\section{INTRODUCTION}

The introduction of Helfrich's expression for the curvature free energy ${ }^{1}$ presented an important step in the theoretical understanding of complex interfaces. In terms of the two elasticity or rigidity constants, $k$ and $\bar{k}$, as well as the radius of spontaneous curvature $R_{0}$, the Helfrich free energy was able to describe the shape, fluctuations and free energy of interfaces covered by a monolayer or bilayer of surface active molecules such as (in)soluble surfactants and lipids. The Helfrich energy has, therefore, been used to describe membranes, vesicles, microemulsion systems and to calculate their respective phase diagrams. ${ }^{2}$ Seifert and Lipowsky ${ }^{3,4}$ were the first to apply the Helfrich free energy for the description of the shape and free energy of a vesicle adhered to a solid substrate (see Fig. 1). In particular they calculated phase diagrams of the unbinding transition in which the vesicle desorbs from a substrate. Unfortunately, the differential equations describing the shape of the adhered vesicle cannot, in general, be solved analytically so that Seifert and Lipowsky had to resort to solving these shape equations numerically. ${ }^{3-5}$ The large number of parameters such as the prescribed surface area $A$, vesicle volume $V$, the rigidity constants as well as the adhesion energy makes numerical work rather tedious, however, and the need arises for limiting analytical results. In this article we derive such a limiting solution by calculating the shape and free energy of a vesicle adhered to a substrate, or of two microemulsion droplets forming a dimer, under the condition that the rigidity con-

a)Electronic mail: edgar@chemfcb.leidenuniv.nl stant $k$ is small. ${ }^{6}$ Specifically, the length $(k / \sigma)^{1 / 2}$ constructed from the rigidity constant and surface tension $\sigma$, will be assumed to be small compared to the typical size of the system, $(k / \sigma)^{1 / 2} \ll R$. The leading order contribution in small $k$ to the free energy of the adhered vesicle is calculated and formulas are presented describing vesicle adhesion under different conditions of constant volume and constant pressure. Since the free energy in the case of a single vesicle adhered to a substrate can be calculated numerically exact, the comparison with our formulas performed in Sec. II is done mainly as a numerical check of our results. Our main motivation for the present calculation lies in the application of our formulas to the description of droplet aggregation in microemulsions where such numerical work is difficult due to the large number of parameters involved.

At present, a complete understanding of aggregation processes and shape transformations in microemulsion systems is lacking. ${ }^{6}$ Certain aspects, however, appear to be well understood. It seems well established by, among others, the work of Safran and co-workers, ${ }^{2,7}$ that the curvature energy of the interfacial surfactant layer, as described by the Helfrich free energy, plays an important role in the description of shape fluctuations and the many structural transitions that are encountered. ${ }^{8}$ These include transitions from spherical to cylindrical micelles, ${ }^{9}$ their ordering into crystalline (e.g., hexagonal) arrays, transitions from cylindrical micelles to lamellar liquid crystalline phase, as well as transitions from cylindrical micelles to bicontinuous monolayer phases and from bicontinuous bilayer phases (L3) to lamellar liquid crystalline phases. 


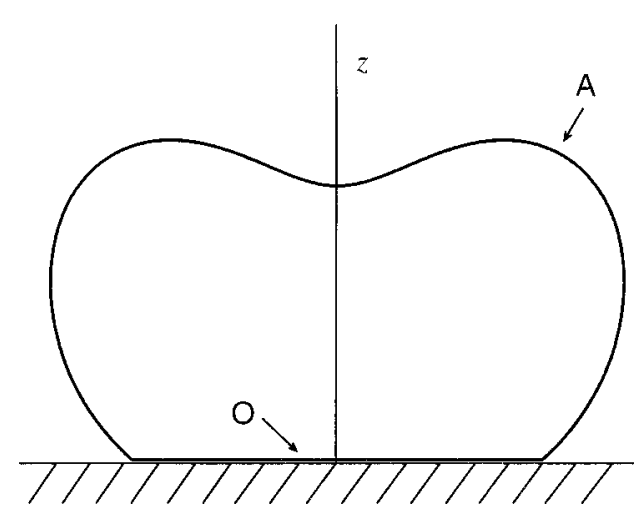

FIG. 1. Vesicle adhered to a substrate located at $z=0, A$ is the surface area of the whole droplet including the surface area $O$ that is in contact with the substrate.

Also observed is a liquid-vaporlike transition (with two coexisting microemulsion droplet phases) which, however, is usually described theoretically in terms of liquid state theories treating the microemulsion droplets as hard spheres or sticky hard spheres. ${ }^{10}$ These theories, therefore, do no take into account the elastic properties of the surfactant layer, nor do they account for the observed temperature direction in which phase separation takes place. Experiments have shown ${ }^{11}$ that aggregation processes (which may ultimately lead to the observed phase separation) in microemulsions occur both with increasing and decreasing temperature depending on the microemulsion system studied (e.g., ionic or nonionic surfactant, water-in-oil or oil-in-water microemulsions). In all cases, however, aggregation takes place in the direction of vanishing spontaneous curvature (e.g., with increasing temperature in water-in-oil microemulsions stabilized by an ionic surfactant). To understand the role played by the spontaneous curvature and to achieve a consistent picture of the various phenomena observed in microemulsion systems, the Helfrich curvature free energy should be included in any description of microemulsion droplet aggregation. ${ }^{12}$

In this work we present the necessary first step in the description of droplet aggregation by calculating the curvature free energy of two microemulsion droplets forming a dimer. One can view the formation of dimers as the onset of the observed aggregation phenomena and the main question that we set ourselves out to answer in this article is whether we are able to understand the formation of dimers in the direction of vanishing spontaneous curvature. The calculation of the dimerization free energy is essentially the same as in the case of vesicle adhesion when instead of a vesicle adhered to a substrate one then describes the adhesion of two microemulsion droplets. For two reasons the small bending rigidity regime seems appropriate for this calculation. First, experimentally it seems well established ${ }^{13}$ that the free microemulsion droplets are spherical or nearly spherical. This can only be the case when the influence of the rigidity constant is small compared to that of the surface tension. Furthermore, as discussed above, it seems that in the description of microemulsion aggregation, the important curvature vari- able is not the rigidity constant, but rather the radius of spontaneous curvature. ${ }^{14}$

The outline of this article is as follows. In Sec. II we introduce the necessary ingredients for the calculation of the shape and free energy of a vesicle adhered to the substrate. The calculation of the leading contribution to the free energy in small $k$ is calculated leaving most of the details of the calculation to the Appendix. At the end of the section explicit formulas for the free energy of the vesicle adhered to a substrate are presented and compared to numerical solutions of the shape equations. In Sec. III we apply the free energy obtained in Sec. II to study the dimerization of microemulsion droplets. The merits and limitations of our calculation are discussed in Sec. IV.

\section{HELFRICH FREE ENERGY}

In this section we concentrate on the problem of a vesicle adhered to a solid substrate (Fig. 1). Different contributions to the free energy describe its shape. The first contribution is the Helfrich free energy ${ }^{1}$ describing the bending energy of the vesicle membrane in terms of the radius of spontaneous curvature $R_{0}$, the rigidity constant associated with bending, $k$, and the rigidity constant associated with Gaussian curvature, $\bar{k}$,

$$
F_{H}=\int d A\left[\sigma-\frac{2 k}{R_{0}} J+\frac{k}{2} J^{2}+\bar{k} K\right],
$$

where $\sigma$ is the surface tension of the planar membrane surface. The above free energy features an integral over the whole surface area, $A$, of the total curvature, $J=1 / R_{1}$ $+1 / R_{2}$ and Gaussian curvature, $K=1 /\left(R_{1} R_{2}\right)$ with $R_{1}$ and $R_{2}$ the principal radii of curvature at a certain point on the surface $A$. The above form for the free energy is the most general form in an expansion up to second order in curvature, and can be viewed as defining the four coefficients $\sigma$, $k / R_{0}, k$, and $\bar{k}$. The rigidity constant associated with Gaussian curvature $\bar{k}$ is a measure of the energy cost for topological changes of the surface. In our case the topology is fixed and the term proportional to $\bar{k}$ can be dropped.

The second contribution to the free energy describes the interaction energy between the vesicle and the substrate. We will assume that the range of the interaction between the substrate and the vesicle is much smaller than the typical dimension of the vesicle, so that the interaction is described by an adhesion energy integrated over the area of contact ${ }^{3}$

$$
F_{s}=\int d O[\Delta \sigma-\sigma],
$$

where $O$ is the area of substrate-vesicle contact and where $\Delta \sigma \equiv \sigma_{s \mathrm{v}}-\sigma_{s}$ is the difference in surface tension of the substrate-vesicle surface and the bare substrate. Since the integration in Eq. (2.1) is over the whole surface area $A$ (including $O$ ), we need to subtract $\sigma$ from $\Delta \sigma$ in the equation above. Later, when we apply our formulas in Sec. III to the case of two microemulsion droplets forming a dimer, we need to replace $\Delta \sigma$ by $\sigma_{b} / 2$, with $\sigma_{b}$ the surface tension of the bilayer formed in between the two droplets. 
The final contribution to the free energy is (minus) the pressure difference $\Delta p$ between the inside and the outside of the vesicle times the volume $V$ of the vesicle

$$
F_{p}=\int d V[-\Delta p]
$$

so that the total free energy reads

$$
\begin{aligned}
\Omega= & \int d A\left[\sigma-\frac{2 k}{R_{0}} J+\frac{k}{2} J^{2}\right] \\
& +\int d O[\Delta \sigma-\sigma]-\Delta p \int d V .
\end{aligned}
$$

The above free energy is minimal when we fix the surface tension $\sigma$ and the pressure difference $\Delta p$. This would not be the case when, for instance, instead of the surface tension, the surface area $A$ is fixed, or when instead of the pressure difference, the volume is fixed. In these examples the free energies to be minimized are $\Omega-\sigma A$ and $\Omega+\Delta p V$, respectively. Below we address the different ensembles in more detail, but for now it suffices to know that in all these different ensembles the functional form of the free energy to be minimized is that given in Eq. (2.4), with either $\sigma$ and $\Delta p$ as fixed constants or as Lagrange multipliers fixing the surface area and volume.

The shape of the vesicle can be determined by functional minimization of $\Omega$ with respect to the shape. This leads to the so-called shape equation ${ }^{4,15}$

$$
\Delta p=\sigma J-\frac{4 k}{R_{0}} K-\frac{k}{2} J^{3}+2 k J K-k \Delta_{s} J,
$$

where $\Delta_{s}$ denotes the surface Laplacian. This equation can also be seen as the generalized Laplace equation since it reduces to the Laplace equation of a sphere $\Delta p=\sigma J$ $=2 \sigma / R$ when one inserts $J=2 / R$, and sets the coefficients $k / R_{0}$ and $k$ equal to zero. The shape equation [Eq. (2.5)] is equal to the shape equation describing the shape of a free vesicle. ${ }^{15}$ The vesicle adhesion energy is only present in the boundary conditions for the curvature at the substrate. They are given $b y^{3}$

$$
\left.\frac{1}{R_{1}}\right|_{\text {substrate }}=0,\left.\quad \frac{1}{R_{2}}\right|_{\text {substrate }}=[2(\sigma-\Delta \sigma) / k]^{(1 / 2)},
$$

where $R_{2}$ is the radius of curvature along the meridians of the vesicle and $R_{1}$ the radius of curvature perpendicular to it. The first equation indicates that when $k \neq 0$, the contact angle with which the vesicle meets the substrate is always equal to zero. Through the second equation, first derived by Seifert and Lipowsky, ${ }^{3}$ the value of $\Delta \sigma$ enters the description of the shape of the adhered vesicle.

Unfortunately, the shape equations cannot be solved analytically in general. In practice one solves the shape equations numerically for given values of $\sigma, k / R_{0}, k, \Delta \sigma$, and $\Delta p$. With the shape of the vesicle thus obtained one is then able to calculate the volume $V$ and area $A$ of the vesicle together with the appropriate free energies. In this way, one can compare the free energy with the free energy of the unbound vesicle and locate unbinding transitions. For a more elaborate discussion we refer to a recent review by Seifert. ${ }^{4}$

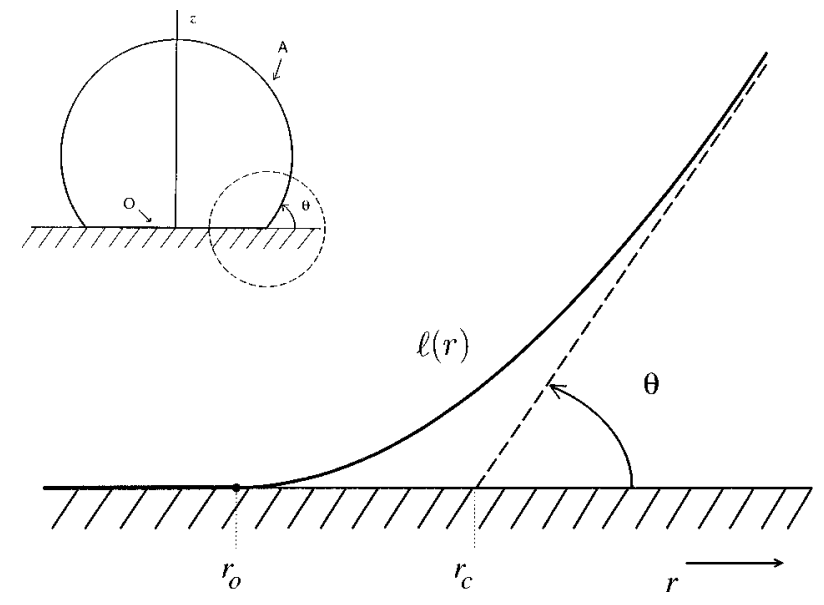

FIG. 2. Height profile $l(r)$, with $r$ the radial distance to the $z$ axis, of the vesicle in the region close to the substrate (see dashed circle in the inset). Lengths are in units of $(k / \sigma)^{1 / 2} \cdot r_{0}$ is the radial distance at which the profile meets the substrate $l\left(r=r_{0}\right)=0$ and $r_{c} \equiv R\left(1-x^{2}\right)^{(1 / 2)}$ is the radius at which the asymptotic spherical-cap-profile (dashed line and inset) meets the substrate with contact angle $\theta$.

The number of parameters makes numerical work rather tedious. Furthermore, it is difficult to gain physical insight into the role played by the different parameters in vesicle unbinding. In order to be able to proceed analytically we need to make certain physically reasonable assumptions. One such assumption, the one that we will adopt in the rest of the article, is to assume that the rigidity constant of bending is small. In particular, the length $(k / \sigma)^{1 / 2}$ will be assumed small compared to the typical size of the vesicle, say $R$. In order to make a systematic expansion in $(k / \sigma)^{1 / 2} / R$, we first assume that $k=0$ and then $k \neq 0$ but small.

\section{A. No bending rigidity}

Before we focus on determining the shape of the adhered vesicle, we first need to address a point of possible confusion. When we set $k=0$, i.e., neglect the second order term in the expansion in the curvature in Eq. (2.1), this does not imply that we will also assume that the coefficient of the first order term, $k / R_{0}$, is equal to zero. Historically, the first order term is defined to contain the rigidity constant, but it is clear that the two coefficients, $k$ and $k / R_{0}$ are independent.

In the absence of bending rigidity, the minimization of the free energy in Eq. (2.4) is easiest done in two steps. First, we note that when $k=0$ the solution of the shape equation in Eq. (2.5) is that of a spherical cap (see the inset of Fig. 2). We know this to be the case when also $k / R_{0}$ is taken to be zero so that the shape equation reduces to the well-known Laplace equation, but also with the spontaneous radius of curvature term present the shape is that of a spherical cap. Second, we insert the spherical-cap-profile, which is fully described by the radius $R$ and contact angle $\theta$, into the expression for the free energy, and then minimize with respect to $R$ and $\theta$.

For the spherical-cap-profile the integration of the free energy over the surface areas $A$ and $O$ and over the volume $V$ in Eqs. (2.1)-(2.3) can be carried out to yield 


$$
\begin{aligned}
F_{H, 0}(R, x)= & \left(\sigma-\frac{4 k}{R_{0}} \frac{1}{R}\right) 2 \pi R^{2}(1+x)+\sigma \pi R^{2}\left(1-x^{2}\right) \\
& -4 \pi \frac{k}{R_{0}} R\left(1-x^{2}\right)^{(1 / 2)} \arccos (x), \\
F_{s, 0}(R, x)= & (\Delta \sigma-\sigma) \pi R^{2}\left(1-x^{2}\right), \\
F_{p, 0}(R, x)= & -\Delta p \frac{\pi}{3} R^{3}\left(2+3 x-x^{3}\right),
\end{aligned}
$$

where we have defined $x \equiv \cos \theta$, and where the subscript 0 stands for the fact that we have set $k=0$. The last term in the expression for $F_{H, 0}(R, x)$ stems from an integration of $J$ across the line where the spherical-cap-profile meets the substrate (see Fig. 2). The presence of this term thus implies that in this case it is not correct to subdivide the integration over $A$ into an integration over the spherical part and the flat part $(O)$.

The total free energy, still as a function of $R$ and $x$, is thus given by

$$
\begin{aligned}
\Omega_{0}(R, x)= & 2 \pi \sigma R^{2}(1+x)+\Delta \sigma \pi R^{2}\left(1-x^{2}\right) \\
& -8 \pi \frac{k}{R_{0}} R(1+x)-4 \pi \frac{k}{R_{0}} R\left(1-x^{2}\right)^{(1 / 2)} \\
& \times \arccos (x)-\Delta p \frac{\pi}{3} R^{3}\left(2+3 x-x^{3}\right) .
\end{aligned}
$$

Finally, $R$ and $x$ are determined by a further minimization of $\Omega_{0}(R, x)$. From $\partial \Omega_{0} / \partial x=0$ and $\partial \Omega_{0} / \partial R=0$ one finds the following set of equations

$$
\begin{aligned}
& \Delta p=\frac{2 \sigma}{R}-\frac{4 k}{R_{0}} \frac{1}{R^{2}}, \\
& \Delta \sigma=\sigma x-\frac{2 k}{R_{0}} \frac{1}{R} x+\frac{2 k}{R_{0}} \frac{1}{R} \frac{\arccos (x)}{\left(1-x^{2}\right)^{(1 / 2)}} .
\end{aligned}
$$

The first equation is the well-known Laplace equation ${ }^{16}$ with a finite size correction originally due to Tolman. ${ }^{17}$ In the work by Tolman the Laplace equation is written as $\Delta p$ $=2 \sigma / R-2 \sigma \delta / R^{2}$, so that the Tolman length $\delta$ is related to the radius of spontaneous curvature via $\sigma \delta=2 k / R_{0} \cdot{ }^{18}$ The second equation determines the value of the contact angle. It reduces to Young's equation ${ }^{19} \sigma_{s \mathrm{v}}=\sigma_{s}+\sigma \cos \theta$ when one inserts $k / R_{0}=0$.

With $R$ and $x$ given by Eqs. (2.9) and (2.10), the free energy $\Omega_{0}$ in the absence of rigidity is now fully determined. Next, we expand around this solution in small $k$.

\section{B. Small bending rigidity}

In order to calculate the leading order contribution to the free energy of the adhered vesicle in small $k$, we need to determine the shape of the vesicle. As shown in the previous section, the shape of the profile is that of a spherical cap when $k=0$ (see the inset of Fig. 2). In the region where the spherical-cap-profile meets the substrate, the first derivative of the height profile is discontinuous so that the curvature $J$, which is related to the second derivative of the height profile, contains a delta function. Thus, when one integrates $J^{2}$, one finds that the curvature energy is infinite. Therefore, for any finite value of $k$ the surface profile has to meet the substrate with zero contact angle as described by the first boundary condition in Eq. (2.6). The result is that deviations of the spherical-cap-profile to leading order in $k$ are located in the region where the spherical-cap-profile meets the substrate (see Fig. 2). The precise shape of the profile near the kink can be determined by minimizing the curvature free energy with the condition that far away from the kink it smoothly crosses over to the spherical-cap-profile with radius $R$ and contact angle $x$. Details of the calculation are presented in the Appendix and here we only list the resulting first order contributions to the free energy (still expressed in terms of the radius $R$ and contact angle $x$ of the asymptotic sphericalcap-profile)

$$
\begin{aligned}
F_{H}(R, x)= & F_{H, 0}(R, x)+2 \pi R(1-x)^{1 / 2}(k \sigma)^{(1 / 2)} 2^{1 / 2} \\
& \times\left[2^{3 / 2}(1+x)^{(1 / 2)}-(3+x)\right] \\
F_{s}(R, x)= & F_{s, 0}(R, x)-2 \pi R(1-x)^{(1 / 2)}\left(\frac{k}{\sigma}\right)^{(1 / 2)} \\
& \times 2^{1 / 2}(\Delta \sigma-\sigma) \\
F_{p}(R, x)= & F_{p, 0}(R, x) .
\end{aligned}
$$

One should keep in mind that $x$ is now not the actual contact angle, which should be zero for any finite value of $k$, but rather the asymptotic contact angle describing the shape of the spherical cap far from the substrate (see Fig. 2).

It is noted from the expressions in Eq. (2.11) that the leading contribution to the free energy is proportional to $k^{1 / 2}$ rather than $k$. Therefore, if we limit our calculation to the leading contribution in $k$, which is $k^{1 / 2}$, we neglect all contributions to the free energy proportional to $k$. In particular we neglect the contribution arising from the integration of the bending energy term $(k / 2) J^{2}$ over regions where the surface is not strongly curved, i.e., far away from the substrate. We also note that to order $k^{1 / 2}$ there is no change in the volume of the vesicle so that $F_{p}=F_{p, 0}$. This result can directly be deduced from Fig. 2 when one keeps in mind that the length scale over which the actual profile differs from the spherical-cap-profile is proportional to $(k / \sigma)^{1 / 2}$ (as shown in the Appendix) so that the associated change in volume is proportional to $\left[(k / \sigma)^{1 / 2}\right]^{2} R$, i.e., proportional to $k$.

We now have all the contributions to the free energy of a single droplet adhered to the substrate expressed in terms of two parameters, the radius $R$ and contact angle $x$. As in Sec. II A, we are only left with the determination of $R$ and $x$ itself via a minimization of the free energy with respect to these parameters. This will be done next considering two ensembles in which such a minimization could take place. First, we consider the constant pressure ensemble and the 
free energy to be minimized is simply $\Omega(\Delta p)=F_{H}+F_{s}$ $+F_{p}$. Second, the constant volume ensemble is considered in which the free energy to be minimized is $F(V) \equiv F_{H}$ $+F_{s}$.

\section{Constant pressure ensemble}

The free energy $\Omega(R, x ; \Delta p)$ is the sum of the free energies in Eq. (2.11)

$$
\begin{aligned}
\Omega(R, x ; \Delta p)= & 2 \pi \sigma R^{2}(1+x)+\pi \Delta \sigma R^{2}\left(1-x^{2}\right)-8 \pi \frac{k}{R_{0}} R(1+x)-4 \pi \frac{k}{R_{0}} R\left(1-x^{2}\right)^{(1 / 2)} \arccos (x) \\
& -\Delta p \frac{\pi}{3} R^{3}\left(2+3 x-x^{3}\right)+2 \pi R(1-x)^{(1 / 2)}(k \sigma)^{(1 / 2)} 2^{1 / 2}\left[2^{3 / 2}(1+x)^{(1 / 2)}-(2+x)-\Delta \sigma / \sigma\right] .
\end{aligned}
$$

The above equation is minimized with respect to $R$ and $x$ yielding the following set of equations:

$$
\begin{aligned}
\Delta p= & \frac{2 \sigma}{R}-\frac{4 k}{R_{0}} \frac{1}{R^{2}}, \\
\Delta \sigma= & \sigma x-\frac{2 k}{R_{0}} \frac{1}{R} x+\frac{2 k}{R_{0}} \frac{1}{R} \frac{\arccos (x)}{\left(1-x^{2}\right)^{(1 / 2)}} \\
& -\frac{(k \sigma)^{(1 / 2)}}{R}\left[4-2^{(3 / 2)}(1+x)^{(1 / 2)}\right],
\end{aligned}
$$

where we have kept terms only to leading order in $k^{1 / 2}$. In comparison with the expressions in Eqs. (2.9) and (2.10) we note that the rigidity constant only appears through the presence of the last term in Eq. (2.14). The Laplace equation with the Tolman correction is therefore unaffected to leading order in $k^{1 / 2}$ by the presence of rigidity.

Next we need to solve $R$ and $x$ from the above set of equations and insert the result in the expression for $\Omega$ in Eq. (2.12). This is done perturbatively in an expansion in $(k / \sigma)^{1 / 2} \ll R$. At the same time we assume that also $k /\left(\sigma R_{0}\right) \ll R$ so that to zeroth order the radius and asymptotic angle are given by $R_{p} \equiv 2 \sigma / \Delta p$ and $x_{0} \equiv \Delta \sigma / \sigma$, respectively [see Eqs. (2.13) and (2.14)]. In terms of the zeroth order radius and contact angle, $R_{p}$ and $x_{0}$, the free energy can then be shown to be equal to

$$
\begin{aligned}
\Omega(\Delta p)= & \frac{\pi}{3} \sigma R_{p}^{2}\left(2+3 x_{0}-x_{0}^{3}\right) \\
& -4 \pi \frac{k}{R_{0}} R_{p}\left(1-x_{0}^{2}\right)^{(1 / 2)} \arccos \left(x_{0}\right) \\
& -8 \pi \frac{k}{R_{0}} R_{p}\left(1+x_{0}\right)+4 \pi R_{p}(k \sigma)^{(1 / 2)} \\
& \times\left(1-x_{0}^{2}\right)^{(1 / 2)}\left[2-2^{(1 / 2)}\left(1+x_{0}\right)^{(1 / 2)}\right] .
\end{aligned}
$$

Before we turn to the constant volume ensemble, we compare the above free energy to the exact free energy obtained by solving the shape equations numerically. As an example we fix $\Delta p / \sigma$ such that $R_{p}=2$, large compared to $(k / \sigma)^{1 / 2}$ for which we take $(k / \sigma)^{1 / 2}=0.1$, in some arbitrary length unit. Furthermore, $\Delta \sigma$ is varied such that $-1<x_{0}<1$ for two different values of the spontaneous radius of curvature, $k /\left(\sigma R_{0}\right)=0$ and $k /\left(\sigma R_{0}\right)=0.05$.
The result is shown in Fig. 3. The circles and squares are the numerical results for $k /\left(\sigma R_{0}\right)=0$ and $k /\left(\sigma R_{0}\right)=0.05$, respectively. The dashed curve $\left(k /\left(\sigma R_{0}\right)=0\right)$ and the dotdashed curve $\left(k /\left(\sigma R_{0}\right)=0\right)$ are the free energy $\Omega_{0}$ found by setting $k=0$ in Eq. (2.15). The solid curve is the full free energy $\Omega$ in Eq. (2.15) for both $k /\left(\sigma R_{0}\right)=0$ and $k /\left(\sigma R_{0}\right)$ $=0.05$. As can be seen, it agrees well with the numerically obtained free energy.

\section{Constant volume ensemble}

The free energy $F(R, x ; V)$ in the constant volume ensemble is the sum of only the first two free energies in Eq. (2.11)

$$
\begin{aligned}
F(R, x ; V)= & 2 \pi \sigma R^{2}(1+x)+\pi \Delta \sigma R^{2}\left(1-x^{2}\right) \\
& -8 \pi \frac{k}{R_{0}} R(1+x)-4 \pi \frac{k}{R_{0}} R\left(1-x^{2}\right)^{1 / 2} \\
& +2 \pi R(1-x)^{(1 / 2)}(k \sigma)^{(1 / 2)} 2^{1 / 2} \\
& \times\left[2^{3 / 2}(1+x)^{(1 / 2)}-(2+x)-\Delta \sigma / \sigma\right] .
\end{aligned}
$$

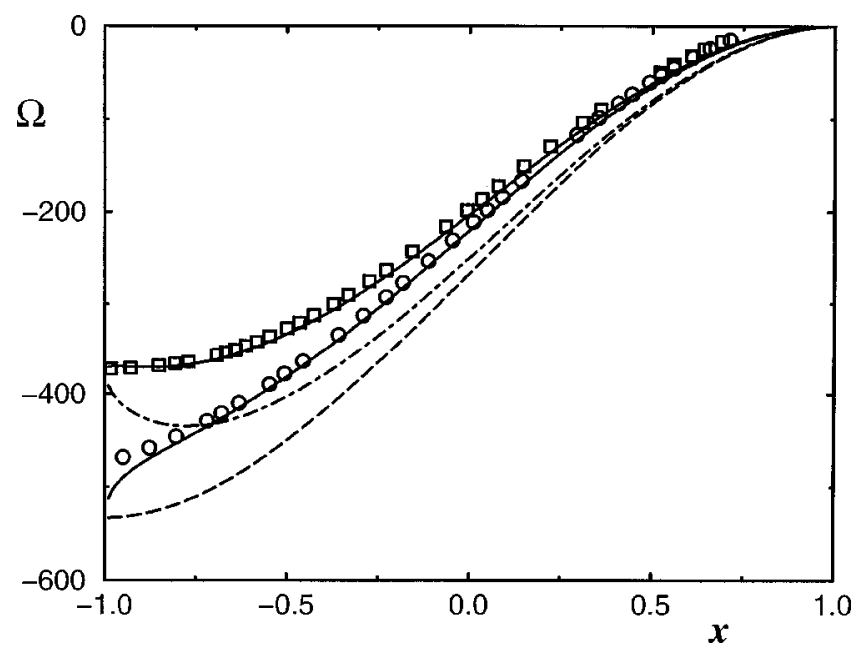

FIG. 3. Free energy, in arbitrary units, as a function of $x_{0}=\Delta \sigma / \sigma$ for fixed pressure difference. The expansion parameter $(k / \sigma)^{1 / 2}=0.1$ in some arbitrary length unit. Circles and squares are the results obtained by numerical solution of the profile for $k /\left(\sigma R_{0}\right)=0$ and $k /\left(\sigma R_{0}\right)=0.05$, respectively. The dashed and the dot-dashed curve are $\Omega$ in Eq. (2.15) with $k=0$ for $k /\left(\sigma R_{0}\right)=0$ and $k /\left(\sigma R_{0}\right)=0.05$, respectively. The solid curves are the full free energy $\Omega$ in Eq. (2.15). 
In the minimization of the above free energy with respect to $R$ and $x$ we have to take into account that now the volume of the vesicle is kept constant $V=(\pi / 3) R^{3}\left(2+3 x-x^{3}\right)$. This is done by adding a term $-\Delta p V$ to the above free energy where $\Delta p$ is now the Lagrange multiplier fixing the vesicle volume. The expression to be minimized is, therefore, exactly equal to $\Omega$ in Eq. (2.12) and the resulting set of equations is again given by Eqs. (2.13) and (2.14). Together with the expression for the volume in terms of $R$ and $x$, these three equations then determine $R, x$, and the Lagrange multiplier $\Delta p$.

Again, we can define the zeroth order radius and (asymptotic) contact angle which are now equal to $R_{V}$ $=\left[3 V /\left(\pi\left(2+3 x_{0}-x_{0}^{3}\right)\right)\right]^{1 / 3}$ and $x_{0} \equiv \Delta \sigma / \sigma$, respectively. In terms of $x_{0}$ and $R_{V}$ the free energy in the constant volume ensemble can then be shown to be equal to

$$
\begin{aligned}
F(V)= & \pi \sigma R_{V}^{2}\left(2+3 x_{0}-x_{0}^{3}\right)-4 \pi \frac{k}{R_{0}} R_{V}\left(1-x_{0}^{2}\right)^{(1 / 2)} \\
& \times \arccos \left(x_{0}\right)-8 \pi \frac{k}{R_{0}} R_{V}\left(1+x_{0}\right) \\
& +4 \pi R_{V}(k \sigma)^{(1 / 2)}\left(1-x_{0}^{2}\right)^{(1 / 2)} \\
& \times\left[2-2^{1 / 2}\left(1+x_{0}\right)^{(1 / 2)}\right] .
\end{aligned}
$$

The above expression for the free energy is quite similar to Eq. (2.15), the only difference being the coefficient of the surface tension term. In particular are the leading order corrections in $k^{(1 / 2)}$ to the free energy in Eqs. (2.15) and (2.17) the same when $R_{p}$ and $R_{V}$ are defined as the zeroth order radius.

One can now also investigate other ensembles for the single vesicle adhered to a substrate such as the ensemble in which besides the volume also the surface area $A$ is kept constant. This, in fact, is the ensemble for which Seifert and Lipowsky ${ }^{3}$ constructed their phase diagrams of the vesicle unbinding transition. In the discussion in Sec. IV we come back to this ensemble but we will first turn our attention to the ensemble appropriate for the determination of the dimerization transition in a microemulsion system.

\section{DIMERIZATION OF MICROEMULSIONS DROPLETS}

In this section we want to discuss the onset of droplet aggregation in microemulsions. With the formulas derived in Sec. II we are able to calculate the free energy for the formation of dimers and construct the phase diagram for droplet-dimer coexistence. Although the calculation of the energy for dimer formation is an important first step, for a full understanding of aggregation in microemulsions the formation of higher aggregates needs to be considered. We come back to this point in the discussion in Sec. IV.

We first need to discuss what is the appropriate ensemble to perform our minimization in. In a single phase microemulsion system two constraints are present: First, the total amount of surfactant is fixed and, second, the total amount of the component inside the microemulsion droplet (e.g., the amount of water when we consider water-in-oil microemulsions) is fixed. These two constraints determine the total amount of surface area available to the surfactant, $A_{\text {tot }}$, and the total amount of droplet volume available to the internal phase, $V_{\text {tot }}$. If $N_{m}$ and $N_{d}$ denote the number of monomeric and dimeric droplets with radius $R_{m}$ and $R_{d}$, respectively, then $V_{\text {tot }}=N_{m} V_{m}+N_{d} V_{d}$ and $A_{\text {tot }}=N_{m} A_{m}+N_{d} A_{d}$ $+N_{d}(\beta-2) O_{d}$. In these expressions the volume of the individual monomer $\left(V_{m}\right)$ and dimer $\left(V_{d}\right)$ are given by

$$
V_{m}=\frac{4 \pi}{3} R_{m}^{3}, \quad V_{d}=\frac{2 \pi}{3} R_{d}^{3}\left(2+3 x-x^{3}\right),
$$

while the surface areas of the individual monomer $\left(A_{m}\right)$ and dimer $\left(A_{d, m}\right.$ of the monolayer and $A_{d, b}$ of the bilayer) are given by

$$
\begin{aligned}
A_{m}=4 & \pi R_{m}^{2}, \\
A_{d, m}= & 4 \pi R_{d}^{2}(1+x)+4 \pi R_{d}(1-x)^{(1 / 2)} \\
& \times\left(\frac{k}{\sigma}\right)^{(1 / 2)} 2^{1 / 2}\left[2^{1 / 2}(1+x)^{(1 / 2)}-1\right], \\
A_{d, b}= & \pi R_{d}^{2}\left(1-x^{2}\right)-2 \pi R_{d}(1-x)^{(1 / 2)}\left(\frac{k}{\sigma}\right)^{(1 / 2)} 2^{1 / 2} .
\end{aligned}
$$

The last two expressions are derived in the Appendix in Eqs. (A22) and (A23), where we have identified $A_{d, m}=2(A$ $-O)$ and $A_{d, b}=O$. Finally, the parameter $\beta$ is the ratio between the number of surfactants per unit area in the bilayer and the number of surfactants per unit area in the monolayer. The value of this parameter is unknown and has to be determined independently from experiments on the monolayer and bilayer surfaces similarly to the surface tension of the bilayer, $\sigma_{b}$, which also has to be determined independently. Since $\beta$ is unknown, we keep it as a variable, so that the phase diagram of the monomer-dimer transition is calculated in terms of $\beta$, but realistically one expects this ratio to be close to two.

In order to investigate the occurrence of a monomer to dimer transition we need to minimize the free energy $F_{m}\left(N_{m}, R_{m}\right)$ of $N_{m}$ monomers and the free energy $F_{d}\left(N_{d}, R_{d}, x\right)$ of $N_{d}$ dimers, with respect to $N_{m}, R_{m}, N_{d}$, $R_{d}$, and $x$, keeping $V_{\text {tot }}$ and $A_{\text {tot }}$ fixed. This is done by adding Lagrange multipliers $(\Delta p, \lambda)$ fixing the total volume $(\Delta p)$ and total surface area $(\lambda)$. The free energy expressions to be minimized, therefore, are

$$
\begin{aligned}
\Omega_{m}\left(N_{m}, R_{m}\right)= & N_{m}\left\{4 \pi \sigma R_{m}^{2}-16 \pi \frac{k}{R_{0}} R_{m}\right. \\
& \left.-\Delta p \frac{4 \pi}{3} R_{m}^{3}+\lambda 4 \pi R_{m}^{2}\right\},
\end{aligned}
$$

for the single microemulsion droplet phase, and 


$$
\begin{aligned}
\Omega_{d}\left(N_{d}, R_{d}, x\right)= & N_{d}\left[4 \pi \sigma R_{d}^{2}(1+x)+\pi \sigma_{b} R_{d}^{2}\left(1-x^{2}\right)-16 \pi \frac{k}{R_{0}} R_{d}(1+x)-8 \pi \frac{k}{R_{0}} R_{d}\left(1-x^{2}\right)^{(1 / 2)} \arccos (x)\right. \\
& -\Delta p \frac{2 \pi}{3} R_{d}^{3}\left(2+3 x-x^{3}\right)+2 \pi R_{d}(1-x)^{(1 / 2)}(k \sigma)^{(1 / 2)} 2^{1 / 2}\left[2^{5 / 2}(1+x)^{(1 / 2)}-2(2+x)-\sigma_{b} / \sigma\right] \\
& +\lambda\left[4 \pi R_{d}^{2}(1+x)+4 \pi R_{d}(1-x)^{(1 / 2)}\left(\frac{k}{\sigma}\right)^{(1 / 2)}\left[2(1+x)^{(1 / 2)}-2^{1 / 2}\right]+\beta \pi R_{d}^{2}\left(1-x^{2}\right)\right. \\
& \left.\left.-2 \beta \pi R_{d}(1-x)^{(1 / 2)}\left(\frac{k}{\sigma}\right)^{(1 / 2)} 2^{1 / 2}\right]\right],
\end{aligned}
$$

for the dimer phase.

Before turning to the minimization of these two free energies, it should be noted that our original reason for neglecting the rigidity constant associated with Gaussian curvature, $\bar{k}$, is now no longer valid, since the topology does change when the number of monomers or dimers varies (as it does). However, since $k$ and $\bar{k}$ are of the same order of magnitude and contributions to the free energy proportional to $k$ are neglected, the Gaussian free energy contribution can again be discarded.

In the case that only single microemulsion droplets are present $\left(N_{d}=0\right)$, the radius $R_{m}$, and number of droplets $N_{m}$ are directly determined by the volume and area constraint. This leads to $R_{m}=3 V_{\text {tot }} / A_{\text {tot }}$ and $N_{m}=A_{\text {tot }}^{3} /\left(36 \pi V_{\text {tot }}^{2}\right)$. The minimizing equations $\partial \Omega_{m} / \partial N_{m}=0$ and $\partial \Omega_{m} / \partial R_{m}=0$ are then only used to determine the values of the (unimportant) Lagrange multipliers $\Delta p$ and $\lambda$. The ratio between the prescribed total volume and surface area defines the important length scale $\omega \equiv V_{\text {tot }} / A_{\text {tot }}$ so that in units of this length scale the radius of the droplet $R_{m}=3$.

Next, we consider the minimization of the free energy in Eq. (3.4) for the dimer phase $\left(N_{m}=0\right)$. Now we have five equations, $\partial \Omega_{d} / \partial N_{d}=0, \partial \Omega_{d} / \partial R_{d}=0$, and $\partial \Omega_{d} / \partial x=0$, together with the total volume and total area constraint. Solving these five equations determines the five unknowns $N_{d}$, $R_{d}, x, \Delta p$, and $\lambda$ in terms of $\beta$, the ratio $\sigma_{b} / \sigma$, the radius of spontaneous curvature $R_{0}$, and $(k / \sigma)^{1 / 2}$. The five equations are again to be solved perturbatively in $k$. First we set $k$ $=0$.

\section{A. No bending rigidity}

The following formula determines the leading order contact angle $x_{0}$ of the dimer phase:

$$
\begin{aligned}
& 3 \omega\left(\sigma_{b}-\beta \sigma\right)\left[4+\beta\left(1-x_{0}\right)\right] \\
& +4 \frac{k}{R_{0}}\left[-12 x_{0}+\beta\left(8+x_{0}-x_{0}^{2}\right)\right. \\
& \left.\quad+\left\{4\left(2 x_{0}^{2}-2 x_{0}-1\right)+3 \beta\left(1-x_{0}\right)\right\} \frac{\arccos \left(x_{0}\right)}{\left(1-x_{0}^{2}\right)^{(1 / 2)}}\right]=0 .
\end{aligned}
$$

In order to determine the free energy $F_{d}$ of the dimers, $x_{0}$ has to be solved from the above equation. As a function of $\sigma_{b} / \sigma, \beta$, and $R_{0}$, different solutions for $x_{0}$ can be found, for which the corresponding free energy can then be calculated (with $N_{d}$ and $R_{d}$ determined from the volume and area constraint)

In Fig. 4, characteristic free energy curves are drawn for $F_{d}$ together with $F_{m}$ (dashed curves) of the monomers for fixed $\sigma_{b} / \sigma$ and $\beta$, but variable $R_{0}$. In Fig. 4(a) the case is

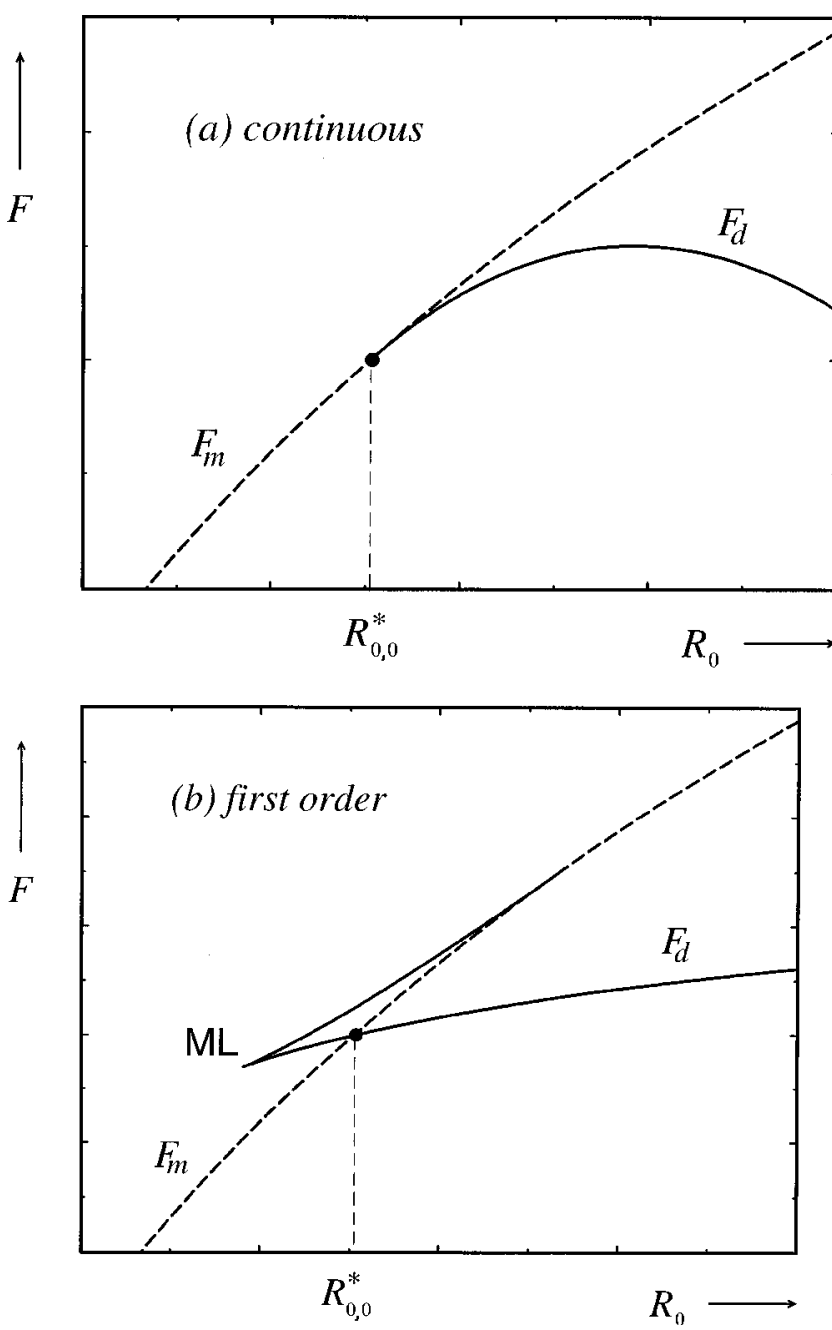

FIG. 4. Characteristic curves for the free energy of the monomers $F_{m}$ and dimers $F_{d}$, as a function of $R_{0}$ when the dimerization transition at $R_{0}$ $=R_{0,0}^{*}$ is continuous (a) or first order (b). In the latter diagram ML denotes the position of the metastable limit beyond which the dimers cease to exist as a metastable phase. 
drawn when only one solution $x_{0}$ exists for $R_{0}>R_{0,0}^{*}$ and none for $R_{0}<R_{0,0}^{*}$. The point $R_{0}=R_{0,0}^{*}$ denotes the location of the dimerization transition, since for $R_{0}<R_{0,0}^{*}$ only monomers are present while for $R_{0}>R_{0,0}^{*}$ the dimers have a lower free energy than the monomers (the $F_{d}$ curve is below the $F_{m}$ curve) and will therefore be energetically preferred. The additional subscript 0 to $R_{0}^{*}$ denotes the fact that the dimerization transition is calculated with $k=0$.

In Fig. 4(a) the dimerization transition is continuous since the free energy curve smoothly crosses over at $R_{0}$ $=R_{0,0}^{*}$. The reason that the dimerization transition is continuous is that at $R_{0}=R_{0,0}^{*}$ the solution for the contact angle of Eq. (3.5) is $x_{0}^{*}=1$, i.e., the contact angle is zero and the shape of the dimer is that of two touching spheres. This implies that the shape and, therefore, the corresponding energy is also "continuous." The value of $R_{0,0}^{*}$ in the case that the transition is continuous can, therefore, easily be determined by setting $x_{0}=1$ into the above equation. One finds

$$
\frac{k}{R_{0,0}^{*}}=\frac{3 \omega}{8} \frac{\beta \sigma-\sigma_{b}}{\beta-2} .
$$

In Fig. 4(b) the case is drawn where as a function of decreasing $R_{0}$ first only one solution for $x_{0}$ to Eq. (3.5) exists, then two solutions and beyond a certain point labeled "ML," the metastable limit, no solutions exist. In this case the dimerization transition is a first order transition: the curve of lowest free energy ( $F=F_{m}$ when $R_{0}<R_{0,0}^{*}$ and $F$ $=F_{d}$ when $R_{0}>R_{0,0}^{*}$ ) displays a discontinuous first derivative at $R_{0}=R_{0,0}^{*}$. The reason is that at $R_{0}=R_{0,0}^{*}$ the solution for the contact angle of Eq. (3.5) is $x_{0}^{*} \neq 1$, so that the shape is "discontinuous." The location of the first order dimerization transition is determined by the requirement that at $R_{0}$ $=R_{0,0}^{*}$ we have that $F_{d}=F_{m}$. One can show that $x_{0}^{*}$ and $R_{0,0}^{*}$ are determined by the following set of equations:

$$
\begin{aligned}
4 x_{0}^{*} & -\beta x_{0}^{*}\left(1-x_{0}^{*}\right)+\beta^{2}\left(1-x_{0}^{*}\right)-\left[4\left[1-x_{0}^{*}+\left(x_{0}^{*}\right)^{2}\right]\right. \\
+ & \left.3 \beta\left(1-x_{0}^{*}\right)\right] \frac{\arccos \left(x_{0}^{*}\right)}{\left[1-\left(x_{0}^{*}\right)^{2}\right]^{(1 / 2)}}=0
\end{aligned}
$$

and

$$
\begin{aligned}
& 4 \frac{k}{R_{0,0}^{*}}\left[-8 x_{0}^{*}+8 \beta+\beta^{2}\left(1-x_{0}^{*}\right)-4\left(2-x_{0}^{*}\right)\right. \\
& \left.\quad \times\left(1+x_{0}^{*}\right) \frac{\arccos \left(x_{0}^{*}\right)}{\left[1-\left(x_{0}^{*}\right)^{2}\right]^{(1 / 2)}}\right]+3 \omega\left(\sigma_{b}-\beta \sigma\right) \\
& \quad \times\left[4+\beta\left(1-x_{0}^{*}\right)\right]=0 .
\end{aligned}
$$

For given value of $\beta$ the first equation [Eq. (3.7)] can be solved to determine $x_{0}^{*}$ which can then be inserted into the second equation [Eq. (3.8)] to determine $R_{0,0}^{*}$. The fact that Eq. (3.7) is independent of $\sigma_{b} / \sigma$ implies that the border between the first order and continuous dimerization is also independent of $\sigma_{b} / \sigma$. One can show that Eq. (3.7) has one or no solutions when $\beta<\beta_{c}$ (the transition is continuous) and two, one or no solutions when $\beta>\beta_{c}$ (the transition is first order), with $\beta_{c}=2+\frac{4}{3} \sqrt{ } 3=4.30 \cdots$.

The results are summarized in Fig. 5 where the phase diagram of the dimerization transition is drawn (solid curve) as a function of the dimensionless spontaneous curvature $C_{0} \equiv k /\left(\omega \sigma R_{0}\right)$ and $\beta$, with $\sigma_{b} / \sigma=1$. From the above analysis we have that the dimerization transition is continuous when $\beta<\beta_{c}$ [with $R_{0,0}^{*}$ determined by Eq. (3.6)] and first order when $\beta>\beta_{c}$ (with $R_{0,0}^{*}$ determined by Eqs. (3.7) and (3.8)].

\section{B. Small bending rigidity}

Next we include rigidity and expand the radius of spontaneous curvature at the transition in $k^{1 / 2}: k / R_{0}^{*}=k / R_{0,0}^{*}$ $+\left(k / \omega^{2} \sigma\right)^{1 / 2} k / R_{0,1}^{*}$. It turns out that the presence of rigidity does not affect the location of the continuous transition to leading order in $k^{1 / 2}, k / R_{0,1}^{*}=0$. It can furthermore be shown that the leading order contribution $k / R_{0,1}^{*}$ for the first order transition is determined by the following equation in terms of $x_{0}^{*}$ and $k / R_{0,0}^{*}$

$$
\begin{aligned}
& 3 \frac{k}{R_{0,1}^{*}}\left(1-x_{0}^{*}\right)\left[4+\beta\left(1-x_{0}^{*}\right)\right]^{2}\left[-8 x_{0}^{*}+8 \beta+\beta^{2}\left(1-x_{0}^{*}\right)-4\left(2-x_{0}^{*}\right)\left(1+x_{0}^{*}\right) \frac{\arccos \left(x_{0}^{*}\right)}{\left[1-\left(x_{0}^{*}\right)^{2}\right]^{(1 / 2)}}\right] \\
& \quad+32 \frac{k}{R_{0,0}^{*}}\left(2-x_{0}^{*}\right)^{2}\left(1+x_{0}^{*}\right)\left(1-x_{0}^{*}\right)^{(1 / 2)}\left[4\left(1+x_{0}^{*}\right)^{(1 / 2)}-2^{3 / 2}-2^{1 / 2} \beta\right]\left[2+\left(1-x_{0}^{*}\right) \frac{\arccos \left(x_{0}^{*}\right)}{\left[1-\left(x_{0}^{*}\right)^{2}\right]^{(1 / 2)}}\right] \\
& \quad-6 \omega\left(\sigma_{b}-\beta \sigma\right)\left(2-x_{0}^{*}\right)\left[1-\left(x_{0}^{*}\right)^{2}\right]^{(1 / 2)}\left[4+\beta\left(1-x_{0}^{*}\right)\right]\left[2^{1 / 2}\left(1+x_{0}^{*}\right)^{(1 / 2)}+2\left(1-x_{0}^{*}\right)\right] \\
& \quad+6 \omega \sigma\left(2-x_{0}^{*}\right)\left[1-\left(x_{0}^{*}\right)^{2}\right]^{(1 / 2)}\left[4+\beta\left(1-x_{0}^{*}\right)\right]^{2}\left[2-2^{1 / 2}\left(1+x_{0}^{*}\right)^{(1 / 2)}\right]=0 .
\end{aligned}
$$

The presence of rigidity shifts the first order transition to lower $C_{0}$ as illustrated by the dashed curve in Fig. 5 which was calculated taking $(k / \sigma)^{1 / 2}=0.1 \omega$.

One observes from Fig. 5 that the transition from mono- mers to dimers indeed occurs with decreasing $1 / R_{0}$ (increasing $\omega$ ). Keeping in mind the assumptions made in our calculation, this seems to proof that curvature energy is the driving force behind the attraction between droplets that 


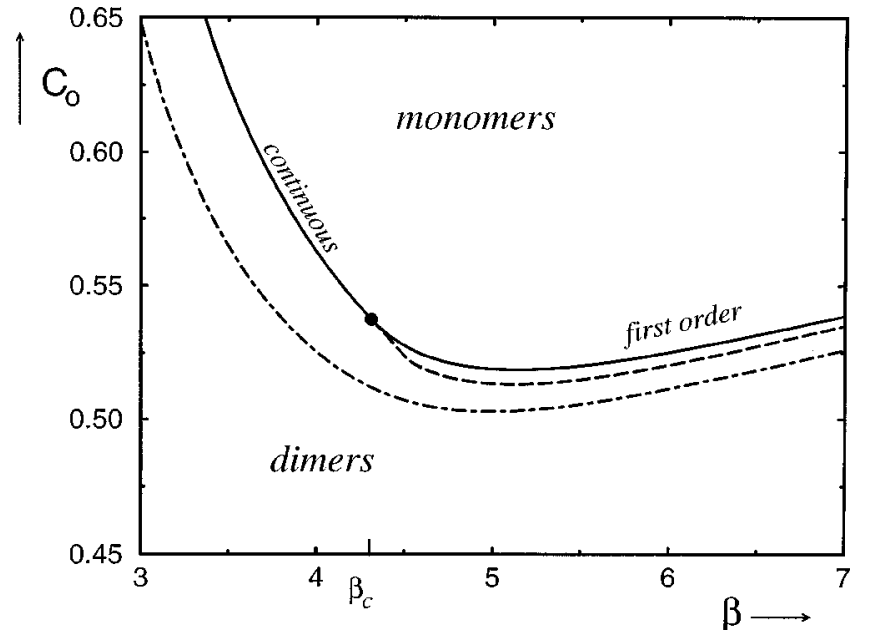

FIG. 5. Phase diagram of the dimerization transition (solid curve) as a function of the reduced spontaneous curvature $C_{0} \equiv k /\left(\omega \sigma R_{0}\right)$ and $\beta$, for $\sigma_{b} / \sigma=1$. The dimerization transition is continuous (second order) when $\beta<\beta_{c}$ and first order when $\beta>\beta_{c}$. The presence of rigidity shifts the first order transition to lower $C_{0}$ as illustrated by the dashed curve which was calculated with $(k / \sigma)^{1 / 2}=0.1 \omega$. The presence of mixing entropy shifts the dimerization transition to lower $C_{0}$ as illustrated by the dot-dashed curve which was calculated with $k_{B} T / \sigma=0.5 \omega^{2}$ and $\phi=0.1$.

ultimately may lead to phase separation. We now show that this result is unaffected by the presence of mixing entropy which we discuss next.

\section{Entropy of mixing}

Until now we have concerned ourselves with the change in curvature energy associated with dimer formation and neglected the loss of entropy associated with dimerization. We discuss qualitatively the influence of entropy by adding a Flory-Huggins-type free energy to the curvature free energy. For the single droplets we add

$$
F_{m, e n t}=\frac{k_{B} T}{\mathrm{v}_{0, m}} V[\phi \ln (\phi)-\phi],
$$

and for the dimers

$$
F_{d, \text { ent }}=\frac{k_{B} T}{\mathrm{v}_{0, d}} V\left[\frac{\phi}{2} \ln \left(\frac{\phi}{2}\right)-\phi\right],
$$

where $k_{B}$ is Boltzmann's constant, $T$ the absolute temperature, $V$ the total available volume to the microemulsion, and $\phi \equiv V_{\text {tot }} / V \ll 1$ is the volume fraction taken in by the microemulsion droplets. The volume $\mathrm{v}_{0}$ is the volume of the unit cell of the Flory-Huggins lattice. Its precise value in microemulsion systems has been subject of intense debate in the literature. ${ }^{20,21}$ Since we are only interested in the qualitative influence of entropy on the dimerization transition, we will not go into this debate and set $\mathrm{v}_{0}$ equal to the volume of the individual microemulsion droplet. We thus have that $\mathrm{v}_{0, m}$ $=(4 \pi / 3) R_{m}^{3}$ for the single droplets and $\mathrm{v}_{0, d}=(\pi / 3)(2+3 x$ $\left.-x^{3}\right) R_{d}^{3}$ for the dimers. Furthermore, we limit our calculation to the case with no bending rigidity, $k=0$.

With these approximations the dimerization transition can be calculated by the minimization of the free energy as before. A typical result is shown as the dot-dashed curve in
Fig. 5 which was calculated with $k_{B} T / \sigma=0.5 \omega^{2}$ and $\phi$ $=0.1$. The presence of mixing entropy is seen to have two effects on the dimerization transition. First, the dimerization transition is shifted to lower $C_{0}$ because the addition of entropy will disfavor the formation of dimers. Second, the transition is now always a first order transition (although weakly first order when $\beta<\beta_{c}$ ). The reason is that, although the curvature energy of two separated microemulsion droplets is the same as when they form a dimer of two touching spheres $(x=1)$, the entropic free energy of these two configurations is always different.

Assuming $k_{B} T /\left(\omega^{2} \sigma\right) \ll 1$ we calculated the leading order shift in the location of the continuous dimerization transition $\left(\beta<\beta_{c}\right)$ with the above form of the entropy. It can be shown that the dimerization transition is given by

$$
\begin{aligned}
\frac{k}{R_{0}^{*}}= & \frac{3 \omega}{8} \frac{\beta \sigma-\sigma_{b}}{\beta-2}-\frac{1}{48}\left[\frac{3 k_{B} T}{\pi} \frac{\beta \sigma-\sigma_{b}}{(\beta-2)^{3}}\right. \\
& \left.\times\left(4+12 \beta-3 \beta^{2}\right) \ln \left(\frac{1}{2 \phi}\right)\right]^{1 / 2} .
\end{aligned}
$$

The leading order contribution due to the presence of entropy to the location of the dimerization is negative and scales as $\left[k_{B} T \ln (1 / 2 \phi)\right]^{1 / 2}$.

\section{DISCUSSION}

In this section we want to discuss our results in the context of the assumptions made throughout this article.

We first focus on the interaction between the vesicle and the substrate (or between two microemulsion droplets). In general, the vesicle-substrate interaction potential $V(l)$ will exhibit a hard-core repulsion at short distances, a minimum at some characteristic distance $l_{\min }$, and decay to zero at large distances. The value of the interaction potential at $l$ $=l_{\text {min }}$ is related to $\Delta \sigma$ via $V\left(l_{\min }\right)=\Delta \sigma-\sigma$ [see Eq. (2.2)]. In this article it was assumed throughout that the typical range of the interaction between the vesicle and the substrate, $l_{\min }$, is much smaller than the dimension of the vesicle (droplet). This meant that the interaction potential could be approximated by a delta function located at the substrate [Eq. (2.2)]. Since the typical size of a vesicle lies in the micrometer range, this assumption seems to be quite reasonable, but in the case of microemulsion droplets the typical size is in the nanometer range and the assumption might become questionable. Furthermore, in the context of the expansion made in terms of the length parameter $(k / \sigma)^{1 / 2}$, it was implicitly assumed in our calculations that the interaction range is also much smaller than $(k / \sigma)^{1 / 2}\left(l_{\text {min }} \ll(k / \sigma)^{1 / 2} \ll R\right)$. In order to go beyond this condition and consider the situation where $l_{\text {min }} \approx(k / \sigma)^{1 / 2}$, it seems difficult to avoid having to solve the shape equations numerically, using the full shape of the substrate-vesicle interaction potential $V(l)$.

However, in the opposite situation when $(k / \sigma)^{1 / 2} \ll l_{\text {min }}$ $\ll R$ the precise shape of the interaction potential can be taken into account via the addition of a line tension term to the free energy. In this regime, the term proportional to $k^{1 / 2}$ in the free energy expression Eq. (2.12) is replaced by a line 
tension free energy $F_{\tau}$ which is proportional to the length of the line where the vesicle profile meets the substrate

$$
F_{\tau}(R, x)=2 \pi R\left(1-x^{2}\right)^{(1 / 2)} \tau .
$$

The value of the proportionality constant, the line tension $\tau$, is determined by the precise shape of the interaction potential between $l=l_{\min }$ and $l=\infty^{22}$

$$
\begin{aligned}
\tau= & \int_{l_{\min }}^{\infty} d l\left[\left\{2 \sigma \Delta V(l)-[\Delta V(l)]^{2}\right\}^{(1 / 2)}\right. \\
& \left.-\left\{2 \sigma E-E^{2}\right\}^{(1 / 2)}\right],
\end{aligned}
$$

where we have defined $\Delta V(l) \equiv V(l)-V\left(l_{\min }\right)=V(l)+\sigma$ $-\Delta \sigma$ and $E \equiv-V\left(l_{\min }\right)=\sigma-\Delta \sigma$. Using the expression for $F_{\tau}(R, x)$ in Eq. (4.1), the radius $R$ and contact angle $x$ can again be determined by minimizing the free energy with respect to $R$ and $x$.

In this context we would like to mention recent work by Fletcher and Petsev ${ }^{23}$ on the aggregation of microemulsion droplets. They too took into account the curvature energy and considered the influence of the full shape of the interaction potential. However, the way in which the curvature energy was taken into account via an increased droplet deformability with vanishing spontaneous curvature, differs from our analysis.

The next important assumption that was made in this article, is the assumption of small bending rigidity, $(k / \sigma)^{1 / 2}$ $\ll R$. As mentioned before, this assumption is partly made to avoid having to resort to numerical work, and partly because it is in line with the experimental findings concerning microemulsion droplets. In the case of vesicle adhesion, neglecting the curvature terms proportional to $k$ has important consequences, however. This is best illustrated by the calculation of the vesicle unbinding transition in the constant volume, constant surface area ensemble. In this ensemble the radius $R$ and contact angle $x$ of the adhered vesicle are simply determined by the geometrical constraints of total volume $V$ and total surface area $A$ [given in Sec. II D and in Eq. (A23), respectively]. For example, to zeroth order we thus have that the contact angle $x_{0}$ is determined by the following algebraic equation [see also Eq. (6.23) in Ref. 4]

$$
\frac{V^{2}}{A^{3}}=\frac{\left(2-x_{0}\right)^{2}\left(1+x_{0}\right)}{9 \pi\left(3-x_{0}\right)^{3}}
$$

As a function of $V$ and $A$, the contact angle, the radius of the adhered vesicle and therefore the free energy, can thus be determined. In order to locate the unbinding transition, the free energy then has to be compared to the free energy of the unbound vesicle. With rigidity neglected to order $k$, however, the shape of the unbound vesicle is always spherical, and $V$ and $A$ cannot be varied independently. Fixing the volume of the spherical vesicle necessarily fixes its surface area. Although this problem does not show up in the ensembles considered in Sec. II C and II D, it is clear that important physical aspects in the treatment of vesicle binding are lost by keeping the expansion only to order $k^{1 / 2}$. Nevertheless, analytical expressions as given in Eqs. (2.15) and (2.17) can provide platforms for calculations which cannot be easily carried out numerically.
Further approximations were made when we applied our formulas to study the onset of microemulsion droplet aggregation. When we located the dimerization transition the formation of higher aggregates was completely discarded. The microemulsion droplets were only allowed to aggregate as dimers or to remain as monomers. This assumption will be more or less valid when the droplet concentration is sufficiently low. However, at high concentrations multiple droplet interaction will have to be taken into account, not only for the formation of, possibly deformed, microemulsion droplet aggregates, but also for the formation of completely new structures, such as cylinders.

In the context of all these approximations we summarize our findings. In this work we have presented the important first step towards a fuller understanding of aggregation phenomena in microemulsion systems by calculating the curvature free energy for the formation of dimers. We found that dimerization occurs in the direction of vanishing spontaneous curvature in agreement with experiments. This seems to proof that curvature energy is the driving force behind the attraction between microemulsion droplets that may ultimately lead to phase separation.

\section{ACKNOWLEDGMENTS}

The research of E.M.B. has been made possible by a fellowship of the Royal Netherlands Academy of Arts and Sciences. The work of W.F.C.S. has been supported by the Netherlands Foundation for Chemical Research $(\mathrm{CW})$ in collaboration with the Netherlands Technology Foundation (STW).

\section{APPENDIX: FIRST ORDER CORRECTIONS TO THE FREE ENERGY}

It will be convenient to express the curvatures $1 / R_{1}$ and $1 / R_{2}$ in terms of the surface height profile $l(r)$, with $r$ the radial distance to the $z$ axis (see Fig. 2). Assuming rotational symmetry around the $z$ axis, they are given by

$$
\frac{1}{R_{1}}=\frac{l^{\prime}(r)}{r\left\{1+\left[l^{\prime}(r)\right]^{2}\right\}^{(1 / 2)}}, \quad \frac{1}{R_{2}}=\frac{l^{\prime \prime}(r)}{r\left\{1+\left[l^{\prime}(r)\right]^{2}\right\}^{(3 / 2)}},
$$

where the prime indicates a differentiation with respect to the argument. For notational conveniences we drop the explicit $r$-dependence of the height profile $l(r)$ in the remainder of the Appendix.

We insert the above expressions for the curvature in terms of the surface profile into the Helfrich free energy expression in Eq. (2.1) 


$$
\begin{aligned}
F_{H}[l]= & \sigma \pi r_{0}^{2}+\left(\sigma-\frac{4 k}{R_{0}} \frac{1}{R}+2 k \frac{1}{R^{2}}\right) 2 \pi R^{2}(1+x)+2 \pi \int_{r_{0}}^{\infty} d r\left[r [ 1 + ( l ^ { \prime } ) ^ { 2 } ] ^ { ( 1 / 2 ) } \left\{\sigma-\frac{2 k}{R_{0}}\right.\right. \\
& \left.\times\left(\frac{l^{\prime}}{r\left[1+\left(l^{\prime}\right]^{2}\right)^{(1 / 2)}}+\frac{l^{\prime \prime}}{\left[1+\left(l^{\prime}\right)^{2}\right]^{(3 / 2)}}\right)+\frac{k}{2}\left(\frac{l^{\prime}}{r\left[1+\left(l^{\prime}\right)^{2}\right]^{(1 / 2)}}+\frac{l^{\prime \prime}}{\left[1+\left(l^{\prime}\right)^{2}\right]^{(3 / 2)}}\right)^{2}\right\}-r\left[1+\left(l_{0}^{\prime}\right)^{2}\right]^{(1 / 2)} \Theta\left(r-r_{c}\right) \\
& \times\left\{\sigma-\frac{2 k}{R_{0}}\left(\frac{l_{0}^{\prime}}{r\left[1+\left(l_{0}^{\prime}\right)^{2}\right]^{(1 / 2)}}+\frac{l_{0}^{\prime \prime}}{\left[1+\left(l_{0}^{\prime}\right)^{2}\right]^{(3 / 2)}}\right)+\frac{k}{2}\left(\frac{l_{0}^{\prime}}{r\left[1+\left(l_{0}^{\prime}\right)^{2}\right]^{(1 / 2)}}+\frac{l_{0}^{\prime \prime}}{\left[1+\left(l_{0}^{\prime}\right]^{2}\right)^{(3 / 2)}}\right)^{2}\right\},
\end{aligned}
$$

where $\Theta\left(r-r_{c}\right)$ is the Heaviside-function and where we have defined $r_{c} \equiv R\left(1-x^{2}\right)^{(1 / 2)}$. The first term is the result of integrating the surface tension over the part of the vesicle that is adhered to the substrate. The integration runs from $r$ $=0$ to $r=r_{0}$ defined as the radial distance at which $l(r$ $\left.=r_{0}\right)=0$ (see Fig. 2). The second term results from the integration of the curvature energy over the spherical cap to which the surface profile smoothly crosses over to at large distances from the substrate. This term is subtracted in the last term of Eq. (A2) via the terms involving the height profile $l_{0}$ of the spherical cap. The reason for adding and sub- tracting the curvature energy of the spherical cap is that the integration over $r$ in the last term could be extended to $r$ $=\infty$ where the profile $l(r) \rightarrow l_{0}(r)$, and the integrand goes to zero.

It will turn out that the leading contribution to the free energy due to the presence of rigidity scales as $k^{1 / 2}$ so that to leading order in $k$ we can disregard the term proportional to $k$ in the expression for the curvature energy of the spherical cap in Eq. (A2). Furthermore, the integration over the first term proportional to $k / R_{0}$ cancels with the same term involving the spherical-cap-profile $l_{0}$ so that Eq. (A2) becomes

$$
\begin{aligned}
F_{H}[l]= & \sigma \pi R^{2}\left(1-x^{2}\right)+\left(\sigma-\frac{4 k}{R_{0}} \frac{1}{R}\right) 2 \pi R^{2}(1+x)+2 \pi \int_{r_{0}}^{\infty} d r\left[\sigma r\left\{\left[1+\left(l^{\prime}\right)^{2}\right]^{(1 / 2)}-1\right\}-\frac{2 k}{R_{0}} \frac{r l^{\prime \prime}}{1+\left(l^{\prime}\right)^{2}}+\frac{k}{2} r\left[1+\left(l^{\prime}\right)^{2}\right]^{(1 / 2)}\right. \\
& \times\left(\frac{l^{\prime}}{r\left[1+\left(l^{\prime}\right)^{2}\right]^{(1 / 2)}}+\frac{l^{\prime \prime}}{\left[1+\left(l^{\prime}\right)^{2}\right]^{(3 / 2)}}\right)^{2}-\Theta\left(r-r_{c}\right)\left\{\sigma r\left[\left(1+\left(l_{0}^{\prime}\right)^{2}\right)^{(1 / 2)}-1\right]\right. \\
& \left.\left.-\frac{2 k}{R_{0}} \frac{r l_{0}^{\prime \prime}}{1+\left(l_{0}^{\prime}\right)^{2}}+\frac{k}{2} r\left[1+\left(l_{0}^{\prime}\right)^{2}\right]^{(1 / 2)}\left(\frac{l_{0}^{\prime}}{r\left[1+\left(l_{0}^{\prime}\right)^{2}\right]^{(1 / 2)}}+\frac{l_{0}^{\prime \prime}}{\left[1+\left(l_{0}^{\prime}\right)^{2}\right]^{(3 / 2)}}\right)^{2}\right\}\right],
\end{aligned}
$$

where in addition we have rewritten the surface tension term somewhat.

As noted before, the integrand in the above expression is written in such a way that it goes to zero when $r \rightarrow \infty$. The main contribution is, therefore, located around $r \approx r_{c}=R\left(1-x^{2}\right)^{1 / 2} \propto R$. We can therefore expand $r$ around $r_{c}$ assuming that the length scale over which the height profile $l(r)$ differs from the spherical-cap-profile $l_{0}(r)$ is small compared to $R$. The result is that we can insert $r=r_{c}+\mathcal{O}(1)$ in the above expression and expand in $1 / R$. To leading order one finds

$$
\begin{aligned}
F_{H}[l]= & \sigma \pi R^{2}\left(1-x^{2}\right)+\left(\sigma-\frac{4 k}{R_{0}} \frac{1}{R}\right) 2 \pi R^{2}(1+x)-4 \pi \frac{k}{R_{0}} R\left(1-x^{2}\right)^{(1 / 2)} \arccos (x)+2 \pi R \\
& \times\left(1-x^{2}\right)^{(1 / 2)} \int_{r_{0}}^{\infty} d r\left[\sigma\left\{\left[1+\left(l^{\prime}\right)^{2}\right]^{(1 / 2)}-1\right\}+\frac{k}{2} \frac{\left(l^{\prime \prime}\right)^{2}}{\left[1+\left(l^{\prime}\right)^{2}\right]^{(5 / 2)}}-\Theta\left(r-r_{c}\right) \sigma\left\{\left[1+\left(l_{0}^{\prime}\right)^{2}\right]^{(1 / 2)}-1\right\}\right],
\end{aligned}
$$

where we have carried out the integration of the terms proportional to $k / R_{0}$ to yield the third term in the above expression. Next, we define $\Delta F \equiv F_{H}-F_{H, 0}$ with $F_{H, 0}$ given in Eq. (2.7),

$$
\begin{aligned}
\Delta F[l]= & 2 \pi R\left(1-x^{2}\right)^{(1 / 2)} \int_{r_{0}}^{\infty} d r\left[\sigma\left\{\left[1+\left(l^{\prime}\right)^{2}\right]^{(1 / 2)}-1\right\}\right. \\
& \left.+\frac{k}{2} \frac{\left(l^{\prime \prime}\right)^{2}}{\left[1+\left(l^{\prime}\right)^{2}\right]^{(5 / 2)}}-\Theta\left(r-r_{c}\right) \sigma\left(\frac{1}{x}-1\right)\right],
\end{aligned}
$$

where we have used the fact that in the limit of large $R$, the spherical-cap-profile $l_{0}$ is a straight line with $l_{0}^{\prime}=\tan \theta$ (see Fig. 2). It will be convenient to define the rescaled lengths

$$
\begin{aligned}
& y \equiv\left(\frac{\sigma}{k}\right)^{(1 / 2)}\left(r-r_{0}\right), \quad \Delta y \equiv\left(\frac{\sigma}{k}\right)^{(1 / 2)}\left(r_{c}-r_{0}\right), \\
& f(y) \equiv\left(\frac{\sigma}{k}\right)^{(1 / 2)} l(r),
\end{aligned}
$$

so that the expression for the free energy can be written as 


$$
\begin{aligned}
\Delta F[f(y)]= & 2 \pi R\left(1-x^{2}\right)^{(1 / 2)}(k \sigma)^{(1 / 2)} \\
& \times \int_{0}^{\infty} d y\left[\left\{\left[1+\left(f^{\prime}\right)^{2}\right]^{(1 / 2)}-1\right\}\right. \\
& \left.+\frac{1}{2} \frac{\left(f^{\prime \prime}\right)^{2}}{\left[1+\left(f^{\prime}\right)^{2}\right]^{(5 / 2)}}-\Theta(y-\Delta y) \sigma\left(\frac{1}{x}-1\right)\right] .
\end{aligned}
$$

The Euler-Lagrange equation to the above free energy reads

$$
\begin{gathered}
\frac{f^{\prime \prime}}{\left[1+\left(f^{\prime}\right)^{2}\right]^{(3 / 2)}}-\frac{f^{\prime \prime \prime \prime}}{\left[1+\left(f^{\prime}\right)^{2}\right]^{(5 / 2)}}+10 \frac{f^{\prime} f^{\prime \prime} f^{\prime \prime \prime}}{\left[1+\left(f^{\prime}\right)^{2}\right]^{(7 / 2)}} \\
-15 \frac{\left(f^{\prime \prime}\right)^{3}}{\left[1+\left(f^{\prime}\right)^{2}\right]^{(7 / 2)}}+\frac{35}{2} \frac{\left(f^{\prime \prime}\right)^{3}}{\left[1+\left(f^{\prime}\right)^{2}\right]^{(9 / 2)}}=0 .
\end{gathered}
$$

This can be integrated once to yield

$$
\begin{aligned}
& \frac{f^{\prime}}{\left[1+\left(f^{\prime}\right)^{2}\right]^{(1 / 2)}}-\frac{f^{\prime \prime \prime}}{\left[1+\left(f^{\prime}\right)^{2}\right]^{(5 / 2)}}+\frac{5}{2} \frac{f^{\prime}\left(f^{\prime \prime}\right)^{2}}{\left[1+\left(f^{\prime}\right)^{2}\right]^{(7 / 2)}} \\
& =\text { constant }=\sin \theta,
\end{aligned}
$$

where the integration constant is determined by the boundary condition that the profile approaches $f(y) \rightarrow \tan \theta(y-\Delta y)$ when $y \rightarrow \infty$. Multiplying the above equation by $f^{\prime \prime}$ and integrating once more, yields

$$
\begin{aligned}
& {\left[1+\left(f^{\prime}\right)^{2}\right]^{(1 / 2)}-\frac{1}{2} \frac{\left(f^{\prime \prime}\right)^{2}}{\left[1+\left(f^{\prime}\right)^{2}\right]^{(5 / 2)}}-\sin \theta f^{\prime}} \\
& =\text { constant }=\cos \theta .
\end{aligned}
$$

Defining $z(y) \equiv f^{\prime}(y)$, we are thus finally left with the following first order differential equation:

$$
z^{\prime}=2^{1 / 2}\left(1+z^{2}\right)^{(5 / 4)}\left[\left(1+z^{2}\right)^{(1 / 2)}-\cos \theta-z \sin \theta\right]^{(1 / 2)} .
$$

We now investigate the boundary condition to the above differential equation. The general boundary conditions to the shape equations are given by Eq. (2.6). With the help of Eq. (A1) they are written as $l^{\prime}\left(r_{0}\right)=0$ and $l^{\prime \prime}\left(r_{0}\right)=[2(\sigma$ $-\Delta \sigma) / k]^{1 / 2}$, which in terms of $z(y)$ reduce to $z(0)=0$ and $z^{\prime}(0)=[2(1-\Delta \sigma / \sigma)]^{1 / 2}$. To leading order in $k$, the latter can be written with the help of Young's equation as $z^{\prime}(0)$ $=[2(1-\cos \theta)]^{(1 / 2)}$. One immediately observes that the profile $z(y)$ indeed obeys this boundary condition when one inserts $z(0)=0$ into the above differential equation.

With the help of the other boundary condition $z(0)=0$, the definition of $z(y)$, and the above differential equation, the height profile $f(y)$ is now written in terms of the following integral:

$$
\begin{aligned}
f(y) & =\int_{0}^{y} d y_{1} z\left(y_{1}\right)=\int_{0}^{z(y)} d z_{1} \frac{z_{1}}{z_{1}^{\prime}} \\
& =2^{-(1 / 2)} \int_{0}^{z(y)} d z_{1} \frac{z_{1}\left(1+z_{1}^{2}\right)^{-(5 / 4)}}{\left[\left(1+z_{1}^{2}\right)^{(1 / 2)}-\cos \theta-z_{1} \sin \theta\right]^{(1 / 2)}} .
\end{aligned}
$$

In order to perform the integration, we define the angle $\beta$ by $z_{1} \equiv \tan \beta$ and rewrite Eq. (A12) as

$$
f(z)=\int_{0}^{\arctan z} d \beta \frac{\sin \beta}{[1-\cos \theta \cos \beta-\sin \theta \sin \beta]^{(1 / 2)}} .
$$

Next, we define the angle $\alpha \equiv \theta-\beta$ so that Eq. (A13) becomes

$$
f(z)=\int_{\theta-\arctan z}^{\theta} d \alpha \frac{\sin \theta \cos \alpha-\cos \theta \sin \alpha}{[1-\cos \alpha]^{(1 / 2)}} .
$$

This integration can be carried out to yield

$$
f(z)=\left[\sin \theta \ln \left(\tan \left(\frac{\alpha}{4}\right)\right)+2 \sin \left(\theta-\frac{\alpha}{2}\right)\right]_{\alpha=\theta-\arctan z}^{\alpha=\theta} .
$$

We have now expressed the height profile $f$ in terms of $z$. In order to determine the height profile as a function of the rescaled radial distance $y$, we need also to express $y$ as a function of $z$. The calculation of $y(z)$ is analogous to the calculation of $f(z)$ and one finds

$$
\begin{aligned}
y(z)= & \int_{0}^{z} d z_{1} \frac{1}{z_{1}^{\prime}}=\left[\cos \theta \ln \left(\tan \left(\frac{\alpha}{4}\right)\right)\right. \\
& \left.+2 \cos \left(\theta-\frac{\alpha}{2}\right)\right]_{\alpha=\theta-\arctan z}^{\alpha=\theta},
\end{aligned}
$$

so that the density profile, parameterized by the angle $\alpha$ which runs from 0 to $\theta$, reads

$$
f(y)=\left\{\begin{aligned}
f(\alpha)= & \sin \theta\left[\ln \left(\tan \left(\frac{\theta}{4}\right)\right)-\ln \left(\tan \left(\frac{\alpha}{4}\right)\right)\right] \\
& -2 \sin \left(\theta-\frac{\alpha}{2}\right)+2 \sin \left(\frac{\theta}{2}\right) \\
y(\alpha)= & \cos \theta\left[\ln \left(\tan \left(\frac{\theta}{4}\right)\right)-\ln \left(\tan \left(\frac{\alpha}{4}\right)\right)\right] \\
& -2 \cos \left(\theta-\frac{\alpha}{2}\right)+2 \cos \left(\frac{\theta}{2}\right)
\end{aligned}\right.
$$

Before calculating the free energy $\Delta F_{H}$, we determine the rescaled distance $\Delta y$. It is defined by the asymptotic behavior of the profile $f(y) \rightarrow(y-\Delta y) \tan \theta$ when $y \rightarrow \infty$

$$
\begin{aligned}
\Delta y & =\lim _{y \rightarrow \infty}\left[y-\frac{f(y)}{\tan \theta}\right] \\
& =\lim _{y \rightarrow \infty} \int_{0}^{y} d y_{1}\left[1-\frac{z\left(y_{1}\right)}{\tan \theta}\right]=\int_{0}^{\infty} d y\left[1-\frac{z(y)}{\tan \theta}\right] .
\end{aligned}
$$

Using the differential equation Eq. (A11) for the height profile, one finds, following the same procedure as before, that:

$$
\Delta y=\frac{2^{1 / 2}}{(1+x)^{(1 / 2)}} .
$$

We now return to the calculation of the Helfrich free energy. Inserting the differential equation [Eq. (A10)] into the expression for $\Delta F$ in Eq. (A7), one finds 


$$
\begin{aligned}
\Delta F= & 2 \pi R\left(1-x^{2}\right)^{(1 / 2)}(k \sigma)^{(1 / 2)} \int_{0}^{\infty} d y\left\{2\left[1+\left(f^{\prime}\right)^{2}\right]^{(1 / 2)}\right. \\
& \left.-1-\cos \theta-\sin \theta f^{\prime}-\Theta(y-\Delta y)\left(\frac{1}{\cos \theta}-1\right)\right\} \\
= & 2 \pi R\left(1-x^{2}\right)^{(1 / 2)}(k \sigma)^{(1 / 2)} \int_{0}^{\infty} d y\left\{2\left(1+z^{2}\right)^{(1 / 2)}-1\right. \\
& \left.-\cos \theta+z\left(\frac{1}{\tan \theta}-\frac{1}{\sin \theta}-\sin \theta\right)\right\} .
\end{aligned}
$$

Following the same procedure as before, we write the integration in terms of $z$ instead of $y$ using Eq. (A11), define $z$ $\equiv \tan \beta$ and then define $\alpha \equiv \theta-\beta$. This leaves us with the following integral:

$$
\begin{aligned}
\Delta F= & 2 \pi R\left(1-x^{2}\right)^{(1 / 2)}(k \sigma)^{(1 / 2)} 2^{1 / 2} \\
& \times \int_{0}^{\theta} d \alpha\left\{2(1-\cos \alpha)^{(1 / 2)}-\frac{(1-\cos \theta) \sin \alpha}{\sin \theta(1-\cos \alpha)^{(1 / 2)}}\right\} \\
= & 2 \pi R(1-x)^{(1 / 2)}(k \sigma)^{(1 / 2)} 2^{1 / 2}\left[2^{3 / 2}\right. \\
& \left.\times(1+x)^{(1 / 2)}-(3+x)\right] .
\end{aligned}
$$

This is our final result as presented in Eq. (2.11). In addition to the Helfrich free energy, we need to calculate the adhesion energy $F_{s}$. For this we need to calculate the surface area $O=\pi r_{0}^{2}$. With the definition of $\Delta y$ in Eq. (A6) and the explicit expression for $\Delta y$ in Eq. (A19), we see that it is given by

$$
O=\pi R^{2}\left(1-x^{2}\right)-2 \pi R(1-x)^{(1 / 2)}\left(\frac{k}{\sigma}\right)^{(1 / 2)} 2^{(1 / 2)} .
$$

As a final point we list the result for the calculation of the total surface area of the vesicle $A$

$$
\begin{aligned}
A= & O+2 \pi R^{2}(1+x)+2 \pi \int_{r_{0}}^{\infty} d r\left[r\left\{\left[1+\left(l^{\prime}\right)^{2}\right]^{(1 / 2)}-1\right\}\right. \\
& \left.-\Theta\left(r-r_{c}\right) r\left\{\left[1+\left(l_{0}^{\prime}\right)^{2}\right]^{(1 / 2)}-1\right\}\right] \\
= & \pi R^{2}\left(3+2 x-x^{2}\right)+2 \pi R(1-x)^{(1 / 2)}\left(\frac{k}{\sigma}\right)^{(1 / 2)} 2^{1 / 2}\left[2^{1 / 2}\right. \\
& \left.\times(1+x)^{(1 / 2)}-2\right],
\end{aligned}
$$

which we need for our calculation in Sec. III.
${ }^{1}$ W. Helfrich, Z. Naturforsch. C 28, 693 (1973).

${ }^{2}$ For reviews see Micelles, Membranes, Microemulsions, and Monolayers, edited by W. M. Gelbart, A. Ben-Shaul, and D. Roux (Springer, New York, 1994); Statistical Mechanics of Membranes and Surfaces, edited by D. Nelson, T. Piran, and S. Weinberg (World Scientific, Singapore, 1988); M. Wortis, U. Seifert, K. Berndl, B. Fourcade, M. Rao, and R. Zia, in Dynamical Phenomena at Interfaces, Surfaces and Membranes, edited by D. Beysens, N. Boccara, and G. Forgacs (Nova Science, New York, 1993).

${ }^{3}$ U. Seifert and R. Lipowsky, Phys. Rev. A 42, 4768 (1990); U. Seifert, Z. Phys. B: Condens. Matter 97, 299 (1995); R. Lipowsky and U. Seifert, Langmuir 7, 1867 (1991); U. Seifert, Phys. Rev. Lett. 74, 5060 (1995).

${ }^{4}$ U. Seifert, Adv. Phys. 46, 13 (1997).

${ }^{5}$ The influence of gravity on the shapes of adhered vesicles is discussed in: M. Kraus, U. Seifert, and R. Lipowsky, Europhys. Lett. 32, 431 (1995).

${ }^{6}$ W. F. C. Sager and E. M. Blokhuis, Prog. Colloid Polym. Sci. 110, 258 (1998); E. M. Blokhuis and W. F. C. Sager, J. Chem. Phys. 110, 3148 (1999).

${ }^{7}$ S. A. Safran, Statistical Thermodynamics of Surfaces, Interfaces, and Membranes (Addison-Wesley, Reading, 1994), and references therein.

${ }^{8}$ J. S. Huang, S. T. Milner, B. Farago, and D. Richter, Phys. Rev. Lett. 59, 2600 (1987); D. Andelman, M. E. Cates, D. Roux, and S. A. Safran, J. Chem. Phys. 87, 7229 (1987); M. Borkovec and H.-F. Eicke, Chem. Phys. Lett. 157, 457 (1989); U. Olsson and H. Wennerström, Adv. Colloid Interface Sci. 49, 113 (1994).

${ }^{9}$ O. Glatter, R. Strey, K-V. Schubert, and E. W. Kaler, Ber. Bunsenges. Phys. Chem. 100, 323 (1996); I. S. Barnes, S. T. Hyde, B. W. Ninham, P-J. Derian, M. Drifford, and T. N. Zemb, J. Phys. Chem. 92, 2286 (1988); U. Olsson, U. Würz, and R. Strey, ibid. 97, 4535 (1993).

${ }^{10}$ S. H. Chen, C. Y. Ku, J. Rouch, P. Tartiaglia, C. Cametti, and J. Samseth, J. Phys. IV 3, 143 (1993); S. V. G. Menon, V. K. Kelkar, and C. Manohar, Phys. Rev. A 43, 1130 (1991).

${ }^{11}$ D. Chatenay, W. Urbach, A. M. Cazabat, and D. Langevin, Phys. Rev. Lett. 54, 2253 (1985); S.-H. Chen, S.-L. Chang, and R. Strey, J. Chem. Phys. 93, 1907 (1990); G. J. M. Koper, W. F. C. Sager, J. Smeets, and D. Bedeaux, J. Phys. Chem. 99, 13291 (1995).

${ }^{12}$ The importance of including rigidity in the description of the adhesion of two vesicles was first discussed by J. N. Israelachvili in Intermolecular and Surface Forces (Academic, New York, 1992).

${ }^{13}$ R. Strey, J. Winkler, and L. Magid, J. Phys. Chem. 95, 7502 (1991); see also A. M. Cazabat and B. Hayter, in Physics of Amphiphiles: Micelles, Vesicles and Microemulsions, edited by V. Degiorgio and M. Corti (North Holland, Amsterdam, 1985), pp. 59-88 and 723-753.

${ }^{14}$ See, e.g., the review by R. Strey, Colloid Polym. Sci. 272, 1005 (1994).

${ }^{15}$ H. J. Deuling and W. Helfrich, J. Phys. (France) 37, 1335 (1976); L. Miao, B. Fourcade, M. Rao, M. Wortis, and R. Zia, Phys. Rev. A 43, 6843 (1991)

${ }^{16}$ J. S. Rowlinson and B. Widom, Molecular Theory of Capillarity (Clarendon, Oxford, 1982).

${ }^{17}$ R. C. Tolman, J. Chem. Phys. 17, 333 (1949).

${ }^{18}$ E. M. Blokhuis and D. Bedeaux, J. Chem. Phys. 97, 3576 (1992).

${ }^{19}$ T. Young, Philos. Trans. R. Soc. London 95, 65 (1805).

${ }^{20}$ For a review of this subject see D. C. Morse, Curr. Opin. Colloid Interface Sci. 2, 365 (1997)

${ }^{21}$ H. Reiss, W. K. Kegel, and J. Groenewold, Ber. Bunsenges. Phys. Chem. 100, 279 (1996).

${ }^{22}$ H. T. Dobbs and J. O. Indekeu, Physica A 201, 457 (1993).

${ }^{23}$ P. D. I. Fletcher and D. N. Petsev, J. Chem. Soc., Faraday Trans. 93, 1383 (1997); D. N. Petsev, Physica A 250, 115 (1998). 